\title{
Self-Heating and Polarization Effects in AlGaN/AlN/GaN/AlGaN Based Devices
}

\author{
K. Ahmeda ${ }^{*+1}$, B. Ubochi ${ }^{*}$, K. Kalna ${ }^{*}$, B. Benbakhti ${ }^{+}$, S. J. Duffy ${ }^{+}$, W. Zhang ${ }^{+}$, A. Soltani ${ }^{\sharp \wedge}$ \\ * Nanoelectronic Devices Computational Group, College of Engineering, Swansea University, Swansea, United Kingdom \\ ${ }^{1}$ k.g.f.ahmeda.717828@swansea.ac.uk \\ ${ }^{+}$Department of Electronics and Electrical Engineering, Liverpool John Moores University, Liverpool, United Kingdom \\ \# LN2 - University of Sherbrooke, Sherbrooke, QC Canada \\ IEMN - University of Lille 1, Villeneuve d'Ascq, France
}

\begin{abstract}
The interplay of self-heating and polarization affecting the current is studied in Al 0.32 Ga0.68N/AIN/GaN/Alo.1Ga0.9N Transmission Line Model (TLM) heterostructures with a scaled source-drain distance. The study is based on meticulously calibrated TCAD simulations against I-V experimental characteristics using an electro-thermal model. The electro-thermal simulations show hot-spots at the edge of the drain contact due to a large electric field affecting the device reliability. Due to the applied electrical stress, the total polarization, relative to the $18 \mu \mathrm{m}$ heterostructure, decreases by 7 $\%, 10 \%$ and $17 \%$ during a reduction of the source-to-drain distance to the $12 \mu \mathrm{m}, 8 \mu \mathrm{m}$, and $4 \mu \mathrm{m}$, respectively, as a result of the additional strain induced by electrical stress. This additional stress on source/drain contacts reduces the polarization at the surface as a result of the inverse piezoelectric effect.
\end{abstract}

Keywords-AlGaN/AlN/GaN/AlGaN Heterostructure; SelfHeating; style; Polarization; Electro-Thermal Simulation

\section{INTRODUCTION}

Gallium Nitride (GaN) wide bandgap semiconductor material possesses attractive properties such as a high breakdown electric field of $3.3 \mathrm{MV} / \mathrm{cm}$, a high carrier mobility in two-dimensional electron gas (2DEG) of $2000 \mathrm{~cm}^{2} / \mathrm{Vs}$, a high electron saturation velocity of $2.5 \times 10^{7} \mathrm{~cm} / \mathrm{s}$, a large energy bandgap of $3.4 \mathrm{eV}$, a low relative permittivity of 10.4 , and a high thermal conductivity $(k)$ of $130 \mathrm{Wm}^{-1} \mathrm{~K}^{-1}[1,2]$. In addition, III-Nitride based semiconductors possess a large polarization, which is the result of an asymmetrical distribution of electron clouds. This property, together with a large band discontinuity at the heterostructure interface, leads to a very effective creation of a 2DEG [3]. Spontaneous and piezoelectric polarizations play a vital role in $\mathrm{AlGaN} / \mathrm{GaN}$ heterostructure based devices.

In this paper, we study the self-heating and polarization behavior of scaled AlGaN/AlN/GaN/AlGaN Transmission Line Model (TLM) heterostructures with low resistive Ohmic contacts aimed for AlGaN/GaN High Electron Mobility Transistors (HEMTs). Understanding the physical transport processes in Ohmic contacts is crucial for the following reasons: (i) reducing the access-resistance and (ii) achieving a higher extrinsic transconductance $(\mathrm{gm})$ [4].

\section{EASE OF USE ALGAN/GAN TLM HETEROSTRUCTURES}

The studied epi-structure was grown by Molecular Beam Epitaxy (MBE) on HP-Si [111] substrate with a thickness of $480 \mu \mathrm{m}$ followed by low-temperature AlN/GaN/AlN nucleation layers, a $1.7 \mu \mathrm{m} \mathrm{Al}_{0.1} \mathrm{Ga}_{0.9} \mathrm{~N}$ back-barrier layer to improve the carrier confinement in the $2 \mathrm{DEG}$, and then by a $15 \mathrm{~nm}$ GaN channel. A $1 \mathrm{~nm}$ AlN spacer has been used to reduce alloy disorder scattering and enhance electron mobility in the channel [5]. This is followed by a $25 \mathrm{~nm}$ undoped $\mathrm{Al}_{0.32} \mathrm{Ga}_{0.68} \mathrm{~N}$ barrier and a $1 \mathrm{~nm} \mathrm{GaN}$ cap layer. The fabrication process flow is similar to that reported in [6]. The spacing between Ohmic contacts varies from $\mathrm{L} 1=4 \mu \mathrm{m}, \mathrm{L} 2=$ $8 \mu \mathrm{m}, \mathrm{L} 3=12 \mu \mathrm{m}$ and $\mathrm{L} 4=18 \mu \mathrm{m} . \mathrm{Ti} / \mathrm{Al} / \mathrm{Ni} / \mathrm{Au}$ $(10 / 200 / 40 / 100 \mathrm{~nm})$ multilayers metallisation scheme was used for the contacts. The contact length (LC) is $50 \mu \mathrm{m}$ for the various distances between contacts, as shown in Fig. 1(a). The energy band diagram overlapped with electron concentration in the heterostructure cross-section is presented in Fig. 1(b). The 2DEG has an electron mobility of $1950 \mathrm{~cm} 2 / \mathrm{Vs}$ at room temperature, an electron sheet density of $1.5 \times 10^{13} \mathrm{~cm}^{-2}$ and an Ohmic contact resistance of $0.3 \Omega . \mathrm{mm}$. The I-V characteristics are measured at DC and dark conditions using Agilent B1500A framework.

\section{Simulation OF THE AlGAN/ALN/GAN/AlGAN TLM STRUCTURES}

The used electro-thermal model combines the twodimensional (2D) Drift-Diffusion (DD) simulations with 2D heat transport model using the commercial tool Atlas-Silvaco. In the calibration step, shown in Fig. 2(a), we have used an electron mobility of $1950 \mathrm{~cm}^{2} / \mathrm{Vs}$ (the same as observed experimentally) and a saturation velocity of $1.9 \times 10^{7} \mathrm{~cm} / \mathrm{s}$ within concentration dependent mobility model [7], in a combination with the nitride specific field dependent mobility model for the high-fields [8]. Additionally, we have included the experimentally measured external resistance at the source/drain (0.3 $\Omega . m m), \quad$ Shockley-Read-Hall (SRH) recombination model and Fermi-Dirac statistics in the simulations. In the simulations without self-heating, the Poisson and continuity equations are solved self-consistently. The simulations with self-heating include a thermal model solved also self-consistently. 


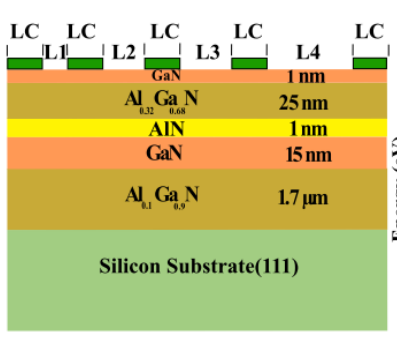

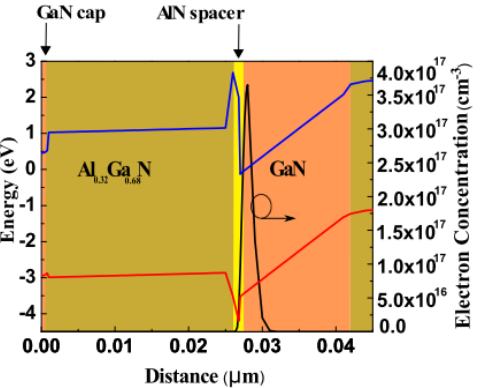

(b)
Fig. 1. (a) Schematic cross section of the GaN based TLM heterostructure and (b) energy band diagram and electron concentration in $2 \mathrm{DEG}$ at equilibrium across.

The thermal mode uses the thermal conductivity approximated by a power function and calibrated to experimental data. The AlGaN/AlN/GaN/AlGaN TLM heterostructures have a p-type doping concentration of $1 \times 10^{16}$ $\mathrm{cm}^{-3}$. In the GaN buffer, we use carbon acceptor traps at an energy of $\mathrm{ET}=\mathrm{EV}+0.9 \mathrm{eV}$ with a density of $1 \times 10^{17} \mathrm{~cm}^{-3}$ which act to reduce a leakage current in the buffer. Additionally, we have used traps corresponding to iron with a concentration of $4 \times 10^{18} \mathrm{~cm}^{-3}$ at $\mathrm{E}_{\mathrm{T}}=\mathrm{EV}+0.6 \mathrm{eV}$ in the $\mathrm{Al}_{0.1} \mathrm{Ga}_{0.9} \mathrm{~N}$ back barrier [9]. The GaN cap donor concentration was set to be $5 \times 10^{20}$ $\mathrm{cm}^{-3}$, which is similar to that reported in [10] with energy level of $\mathrm{E}_{\mathrm{T}}=\mathrm{E}_{\mathrm{C}}-0.5 \mathrm{eV}$ [9].

\section{SElf-HeAting ANd Polarization EFFECTs}

Fig. 2(a) presents the calibrated DD I-V characteristics, of the TLM structure, against the experimental measurements, where self-heating effect is excluded from the simulations. The large difference between the measurement results and the simulations, that occurs for the shortest contact spacing of L1 $=4 \mu \mathrm{m}$ at an applied voltage $\mathrm{V}=15 \mathrm{~V}$, is caused by high electric field value. Fig. 2(b) compares the simulation results obtained from electro-thermal simulations that include selfheating effects. A good I-V calibration has been obtained when including self-heating effects [Fig. 2(b)].

The 2D lattice temperature distribution in the TLM structure of $\mathrm{L} 1=4 \mu \mathrm{m}$ is shown in Fig. 3. The lattice temperature profiles in the 2DEG along the channel for all the structures are presented in Fig. 4. The hot spot is located next to the drain contact for all structures [11]. The shortest structure (L1) exhibits the highest lattice temperature peak of $\sim 566 \mathrm{~K}$, although a smaller voltage is applied than for other structures. Results indicate that there is not much self-heating effect in the TLM structure with largest contact spacing of L4 $=18 \mu \mathrm{m}$. The hot spot remains at the drain side for all TLM structures.

The study on the effect of high electric filed and hot electron on the electrical degradation under applying electrical stress (applied voltage) is studied in details in [12]. It has been demonstrated that an external electrical stress or a voltage, applied on the TLM structure via contacts, can modify the wurtzite crystal structure of III-Nitrides. This phenomenon is known as the inverse piezoelectric effect [13-14]. wurtzite crystal structure of III-Nitrides. This phenomenon is known as the inverse piezoelectric effect [13-14].
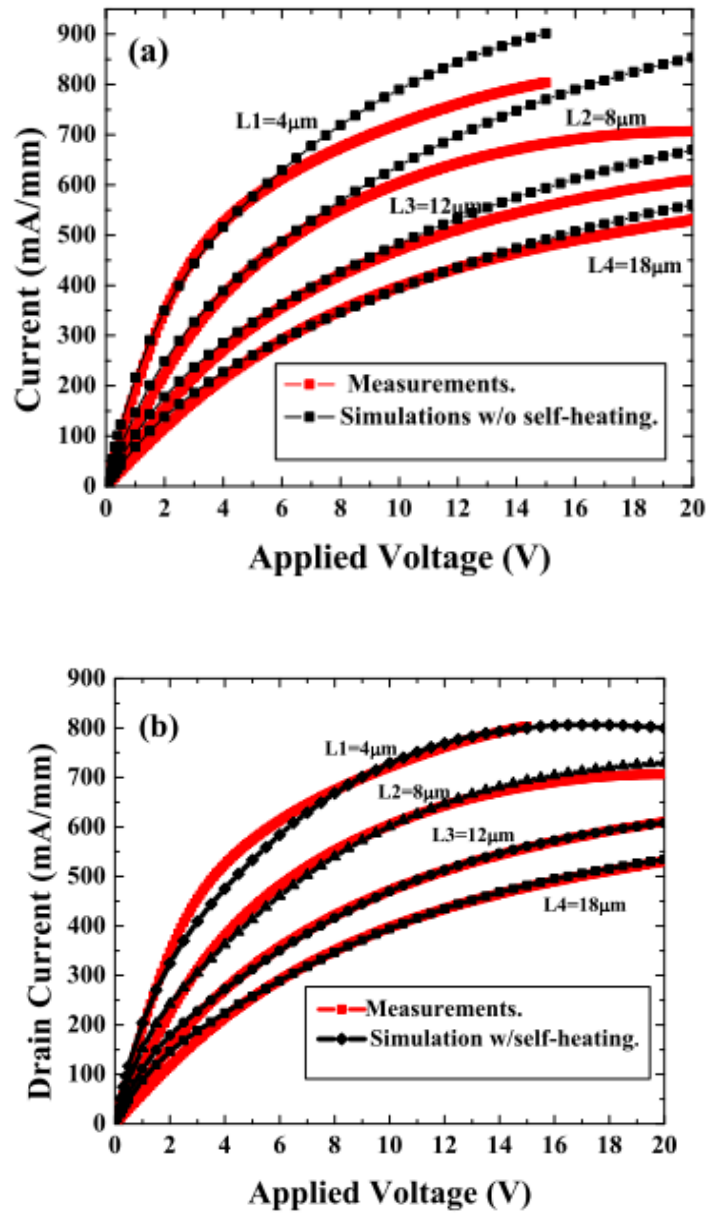

Fig. 2. I-V characteristics of AlGaN/AlN/GaN/AlGaN TLM structures. DD simulations have been calibrated against measured data: (a) I-V characteristics without self-heating effect, (b) electro-thermal simulations of the I-V characteristics including self-heating effect).

To study this phenomenon at different spacing between the contacts, we altered the polarization factor for the TLM structures to mimic the electrical stress that is applied after each measurement and changing the total value of polarization. Fig. 5 illustrates hypothetical I-V characteristics when the polarization factor is fixed at a value calibrated for the TLM structure with L4 $=18 \mu \mathrm{m}$, the largest source-todrain distance. By applying this value on $\mathrm{Ll}=4 \mu \mathrm{m}$, the current increased by $66.8 \%$ in the simulation without selfheating, while it is increased by $44.4 \%$ when including the self-heating. For spacing between contacts of $\mathrm{L} 2=8 \mu \mathrm{m}$, the current increased by $33.5 \%$ without self-heating and $22 \%$ when the self-heating is included. And for L3 $=12 \mu \mathrm{m}$, there is an increase of $17.7 \%$ in the current without self-heating and $13.6 \%$ with self-heating effects.

We assumed that the electrical stress (voltage applied) during the measurements on Ohmic contacts is causing a lattice deformation at the vicinity of the drain, as shown in Fig 6. 


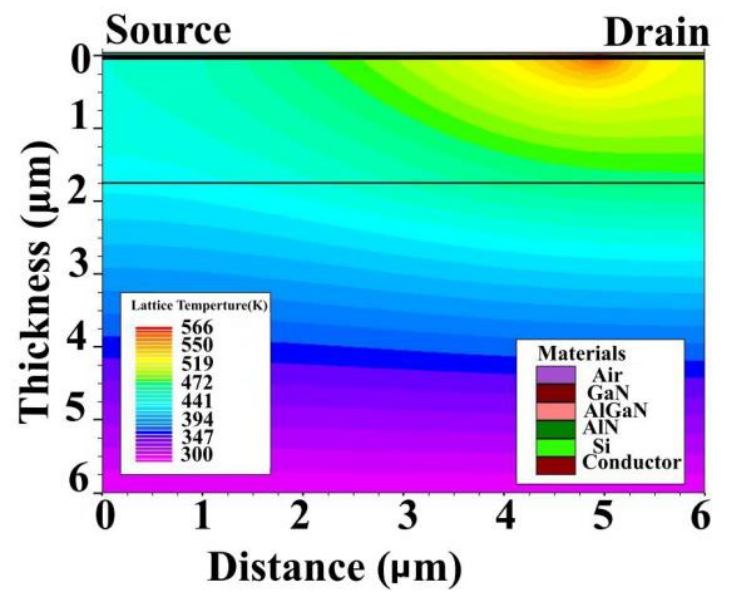

Fig. 3. 2D Lattice temperature distribution in the TLM structure of L1 $=4$ $\mu \mathrm{m}$ at an applied voltage $\mathrm{V}=20 \mathrm{~V}$. The source/drain contact has a length of 1 $\mu \mathrm{m}$ so that the channel starts at $\mathrm{x}=1 \mu \mathrm{m}$ and ends at $\mathrm{x}=5 \mu \mathrm{m}$. The black line is interface between the $\mathrm{Al}_{0.1} \mathrm{Ga}_{0.9} \mathrm{~N}$ back-barrier layer and the silicon substrate.

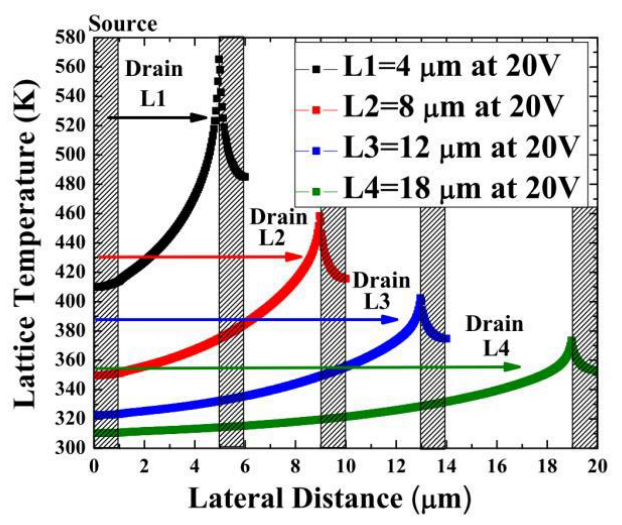

Fig. 4. Lattice temperature profiles in the 2DEG along the channel. The hot spot is located at the drain contact, for all structures. The location of the source and drain contacts are indicated.

The total polarization value decreases when compared to the largest contact of $18 \mu \mathrm{m}$ for $12 \mu \mathrm{m}, 8 \mu \mathrm{m}$, and $4 \mu \mathrm{m}$ by $7 \%$, $10 \%, 17 \%$, respectively. The inverse piezoelectric effect induced decrease in total polarization is caused by the additional stress imposed by the applied electric field on the drain contact. The decrease in total polarization in the heterostructure reduces a $2 \mathrm{DEG}$ in the channel, which would reduce the drain current of a transistor in on-conditions [10$11]$.

\section{CONCLUSION}

We have investigated the interplay of self-heating and polarization in $\mathrm{GaN} / \mathrm{Al}_{0.32} \mathrm{Ga}_{0.68} \mathrm{~N} / \mathrm{AlN} / \mathrm{GaN} / \mathrm{Al} 0.1 \mathrm{Ga} 0.9 \mathrm{~N}$ TLM structures grown on Si-HP (111) substrate. The measured I-V characteristics were simulated via a $2 \mathrm{D}$ electrothermal drift-diffusion model using Fermi-Dirac statistics and the SRH recombination model by commercial tool Atlas by

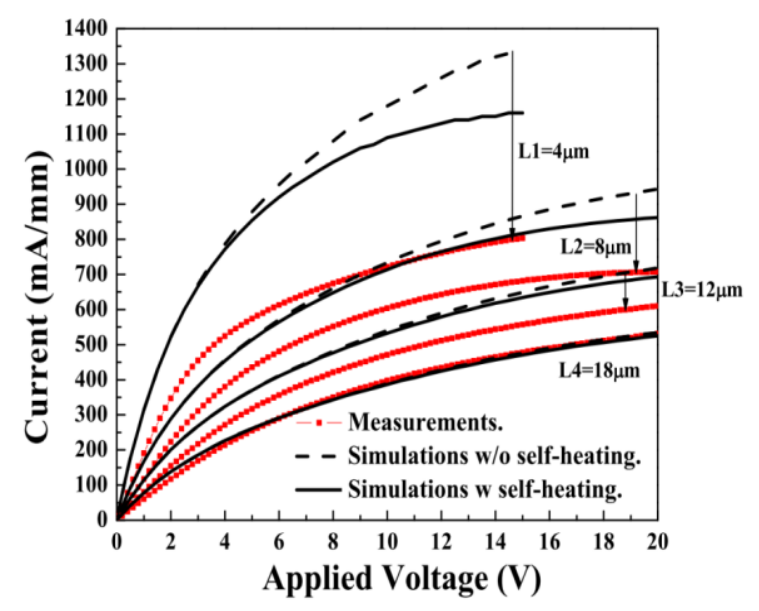

Fig. 5. Measured I-V characteristic of TLM structures (red lines) plotted against the hypothetical low-field calibrated results (black dashed line without self-heating and black line with self-heating) assuming fixed polarization value for the largest structure L4 $=18 \mu \mathrm{m}$. Example of a figure caption. (figure caption)

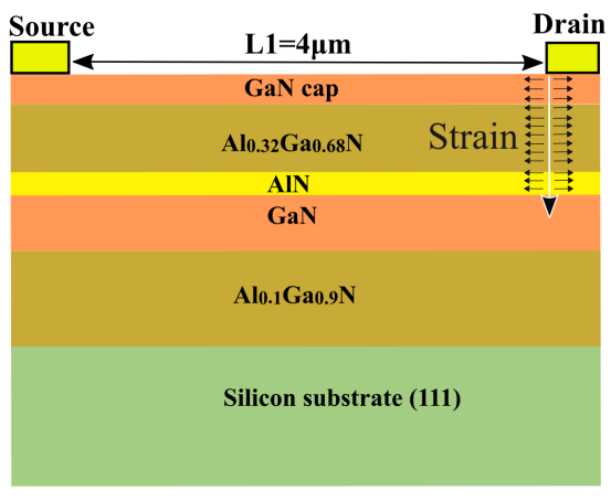

Fig. 6. Schematic diagram of $4 \mu \mathrm{m}$ TLM structure illustrates the strain induces by applied electrical stress.

Silvaco. The electric transport in the drift-diffusion model used mobility model with a low-field mobility of $1950 \mathrm{~cm}^{2} / \mathrm{Vs}$ and a saturation velocity of $1.9 \times 10^{7} \mathrm{~cm} / \mathrm{s}$ assuming the experimental source/drain resistance $(0.3 \Omega . \mathrm{mm})$. Thermal model was employed to study the self-heating effects with the thermal conductivity approximated by a power function and calibrated to experimental data.

We have found that the current becomes soon limited by increase in a lattice temperature up to $13 \%$ (the $4 \mu \mathrm{m}$ structure) with the increase in an applied bias and that this limitation occurs sooner in shorter structures. The maximum temperature $(566 \mathrm{~K})$ was predicted at a vicinity of the drain. In addition, we have observed that, by applying electrical stress (voltage) on the Ohmic contacts, the total polarization value is reduced when compared to the largest contact distance of 18 $\mu \mathrm{m}$ for the $12 \mu \mathrm{m}, 8 \mu \mathrm{m}$, and $4 \mu \mathrm{m}$ TLM structures by $7 \%, 10$ $\%, 17 \%$, respectively. This decrease in the total polarization is due to the inverse piezoelectric effect caused by the additional stress induced by the applied electric field on the contacts, which then changes the total polarization in the 
heterostructure, thus affecting density of a 2DEG in the channel [12-13].

\section{REFERENCES}

[1] R. J. Trew, "High-frequency solid-state electronic devices," IEEE Trans. Electron Devices, vol. 52, 638-649,May 2005

[2] M. Ishida, Y. Uemoto, T. Ueda, T. Tanaka, D. Ueda, "GaN power switching devices," Proc. Int. Power Electronics Conference (IPEC), pp. 1014-1017, 2010.

[3] R. Vetury, N.Q. Zhang, S. Keller, U.K. Mishra, "The impact of surface states on the DC and RF characteristics of AlGaN/GaN HFETs," IEEE Trans. Electron Devices, vol. 48 560-566, 2001.

[4] S. Taking, "AlN/GaN MOS-HEMTs Technology," PhD Thesis, School of Engineering, University Of Glasgow, 2012.

[5] I. P. Smorchkova, L. Chen, T. Mates, L. Shen, S. Heikman, B. Moran, S Keller, S. P. DenBaars, J. S. Speck, and U. K. Mishra, "AlN/GaN and $(\mathrm{Al}, \mathrm{Ga}) \mathrm{N} / \mathrm{AlN} / \mathrm{GaN}$ two-dimensional electron gas structures grown by plasma-assisted molecular-beam epitaxy," J. Appl. Phys., vol. 90, 51965201, 2001.

[6] A. Soltani, J. C. Gerbedoen, Y. Cordier, D. Ducatteau, M. Rousseau, M. Chmielowska, M. Ramdani, and J. C. D. Jaeger, "Power performance of $\mathrm{AlGaN} / \mathrm{GaN}$ High-electron-mobility transistors on (110) silicon substrate at $40 \mathrm{GHz}$," IEEE Electron Device Lett., vol. 34, 490-492, 2013.

[7] S. Bajaj, O. F. Shoron, P. S. Park, S. Krishnamoorthy, F. Akyol, T.-H. Hung, S. Reza, E.M. Chumbes, J. Khurgin, and S. Rajan, "Densitydependent electron transport and precise modeling of GaN high electron mobility transistors," Appl. Phys. Lett., vol. 107, 153504, 2015.

[8] M. Farahmand, C. Garetto, E. Bellotti, K.F. Brennan, M. Goano, E. Ghillino, G. Ghione, J.D. Albrecht, P.P. Ruden, "Monte Carlo simulation of electron transport in the III-nitride wurtzite phase materials system: binaries and ternaries," IEEE Trans. Electron Devices, vol. 48 , 535-542, 2001.

[9] M. J. Uren, J. Möreke, and M. Kuball, "Buffer design to minimize current collapse in GaN/AlGaN HFETs," IEEE Trans. Electron Devices, vol. 59, 3327-3333, 2012.

[10] J. Kuzmik, C. Ostermeier, G. Pozzovivo, B. Basnar, W. Schrenk, J. F. Carlin, M. Gonschorek, E. Feltin, N. Grandjean,Y. Douvry, C. Gaquiere, J. C. De Jaeger, K. Cico, K. Frohlich, J. Skriniarova, J. Kovac, G. Strasser, D. Pogany, and E. Gornik, "Proposal and performance analysis of normally off $\mathrm{n}++\mathrm{GaN} / \mathrm{InAlN} / \mathrm{AlN} / \mathrm{GaN}$ HEMTs with 1-nm-thick InAIN barrier," IEEE Trans. Electron Devices, vol. 57, 2144-2154, 2010.

[11] T. Sadi, R. W. Kelsall, N. J. Pilgrim, "Investigation of self-heating effects in submicrometer GaN/AlGaN HEMTs using an electrothermal Monte Carlo method," IEEE Trans. Electron Devices, vol. 53, 28922899, 2006.

[12] M. Faqir, G. Verzellesi, G. Meneghesso,E. Zanoni, and F. Fantini "Investigation of High-Electric-Field Degradation Effects in AlGaN/GaN HEMTs" IEEE Trans. Electron Devices, vol. 55, no. 7, July 2008

[13] U. Chowdhury, J. L. Jimenez, C. Lee, E. Beam, P. Saunier, T. Balistreri, S.Y. Park, T. Lee, J. Wang, M. J. Kim, J. Joh, J. A. de Alamo, “TEM Observation of crack- and pit-shaped defects in electrically degraded GaN HEMTs," IEEE Electron Device Lett., vol. 29, 1098-1100, 2008.

[14] R. Chu, "Gate-recessed GaN high electron mobility transistors with scaled gate length," Dept. Electrical \& Computer Engineering, University Of California, Santa Barbara, Dec 2008. 